\title{
APPROXIMATION AND OPTIMALITY NECESSARY CONDITIONS IN RELAXED STOCHASTIC CONTROL PROBLEMS
}

\author{
SEÏD BAHLALI, BRAHIM MEZERDI, AND BOUALEM DJEHICHE
}

Received 28 April 2005; Accepted 5 March 2006

We consider a control problem where the state variable is a solution of a stochastic differential equation (SDE) in which the control enters both the drift and the diffusion coefficient. We study the relaxed problem for which admissible controls are measure-valued processes and the state variable is governed by an SDE driven by an orthogonal martingale measure. Under some mild conditions on the coefficients and pathwise uniqueness, we prove that every diffusion process associated to a relaxed control is a strong limit of a sequence of diffusion processes associated to strict controls. As a consequence, we show that the strict and the relaxed control problems have the same value function and that an optimal relaxed control exists. Moreover we derive a maximum principle of the Pontriagin type, extending the well-known Peng stochastic maximum principle to the class of measure-valued controls.

Copyright (C) 2006 Seïd Bahlali et al. This is an open access article distributed under the Creative Commons Attribution License, which permits unrestricted use, distribution, and reproduction in any medium, provided the original work is properly cited.

\section{Introduction}

We are interested in questions of existence, approximation, and optimality of control problems of systems evolving according to the stochastic differential equation

$$
x_{t}=x+\int_{0}^{t} b\left(s, x_{s}, u_{s}\right) d s+\int_{0}^{t} \sigma\left(s, x_{s}, u_{s}\right) d B_{s},
$$

on some filtered probability space $\left(\Omega, \mathscr{F}_{F},\left(\mathscr{F}_{t}\right)_{t}, P\right)$, where $b$ and $\sigma$ are deterministic functions, $\left(B_{t}, t \geq 0\right)$ is a Brownian motion, $x$ is the initial state, and $u_{t}$ stands for the control variable. The expected cost on the time interval $[0,1]$ is of the form

$$
J(u)=E\left[\int_{0}^{1} h\left(t, x_{t}, u_{t}\right) d t+g\left(x_{1}\right)\right] .
$$

The aim of the controller is to optimize this criterion, over the class $\boldsymbol{U}$ of admissible controls, that is, adapted processes with values in some set $\mathbb{A}$, called the action space. $\mathrm{A}$ 
control $u^{*}$ is called optimal if it satifies $J\left(u^{*}\right)=\inf \{J(u), u \in \mathcal{U}\}$. If, moreover, $u^{*}$ is in $u$, it is called strict. Existence of such a strict control or an optimal control in $u$ follows from the convexity of the image of the action space $\mathbb{A}$ by the map $\left(b(t, x, \cdot), \sigma^{2}(t, x, \cdot), h(t, x, \cdot)\right)$ (Filipov-type convexity condition-see $[2,5,9,10,13])$. Without this convexity condition an optimal control does not necessarily exist in $\mathcal{U}$, the set $\mathcal{U}$ not being equipped with a compact topology. The idea is then to introduce a new class $\mathscr{R}$ of admissible controls, in which the controller chooses at time $t$, a probability measure $\mu_{t}(d a)$ on the control set $\mathbb{A}$, rather than an element $u_{t} \in \mathbb{A}$. These are called relaxed controls. It turns out that this class of controls enjoys good topological properties. If $\mu_{t}(d a)=\delta_{u_{t}}(d a)$ is a Dirac measure charging $u_{t}$ for each $t$, then we get a strict control as a special case. Thus the set $U$ of strict controls may be identified as a subset of $\mathscr{R}$ of relaxed controls.

Using compactification techniques, Fleming [7], derived the first existence results of an optimal relaxed control for SDEs with uncontrolled diffusion coefficient. For such systems of SDEs, a maximum principle has been established in Mezerdi and Bahlali [19]. The case of an SDE where the diffusion coefficient depends explicitly on the control variable has been solved by El-Karoui et al. [5], where the optimal relaxed control is shown to be Markovian.

In this paper we establish two main results. We first show that, under a continuity condition of the coefficients and pathwise uniqueness of the controlled equations, each relaxed diffusion process is a strong limit of a sequence of diffusion processes associated with strict controls. The proof of this approximation result is based on Skorokhod's selection theorem, a limit theorem on martingale measures and Mitoma's theorem [20] on tightness of families of martingale measures. As a consequence, we show that the strict and the relaxed control problems have the same value functions, which yields the existence of nearly optimal strict controls. Note that our result improves those of Fleming [7] and Méléard [14], proved under Lipschitz conditions on the coefficients. Using the same techniques, we give an alternative proof for existence of an optimal relaxed control, based on Skorokhod selection theorem. Existence results were first proved using martingale problems by Haussmann [9] and El-Karoui et al. [5]. The second main result of this paper is a maximum principle of the Pontriagin type for relaxed controls, extending the well-known Peng stochastic maximum principle [22] to the class of measure-valued controls. This leads to necessary conditions satisfied by an optimal relaxed control, which exists under general assumptions on the coefficients. The proof is based on Zhou's maximum principle [26], for nearly optimal strict controls and some stability properties of trajectories and adjoint processes with respect to the control variable.

In Section 2, we define the control problem, we are interested in and introduce some notations and auxiliary results to be used in the sequel. Section 3 is devoted to the proof of the main approximation and existence results. Finally, in Section 4, we state and prove a maximum principle for our relaxed control problem.

\section{Formulation of the problem}

2.1. Strict control problem. The systems we wish to control are driven by the following $d$-dimesional stochastic differential equations of diffusion type, defined on some filtered 
probability space $\left(\Omega, \mathscr{F},\left(\mathscr{F}_{t}\right)_{t}, P\right)$ :

$$
d x_{t}=b\left(t, x_{t}, u_{t}\right) d t+\sigma\left(t, x_{t}, u_{t}\right) d B_{t}, \quad x_{0}=x,
$$

where, for each $t \in[0,1]$, the control $u_{t}$ is in the action space $\mathbb{A}$, a compact set in $\mathbb{R}^{n}$, the drift term $b: \mathbb{R}_{+} \times \mathbb{R}^{d} \times \mathbb{A} \rightarrow \mathbb{R}$, and diffusion coefficient $\sigma: \mathbb{R}_{+} \times \mathbb{R}^{d} \times \mathbb{A} \rightarrow \mathbb{R}^{d} \otimes \mathbb{R}^{k}$ are bounded measurable and continuous in $(x, a)$.

The infinitesimal generator, $L$, associated with (2.1), acting on functions $f$ in $C_{b}^{2}\left(\mathbb{R}^{d}\right.$; $\mathbb{R})$, is

$$
L f(t, x, u)=\frac{1}{2} \sum_{i, j}\left(a_{i, j} \frac{\partial^{2} f}{\partial x_{i} \partial x_{j}}\right)(t, x, u)+\sum_{i}\left(b_{i} \frac{\partial f}{\partial x_{i}}\right)(t, x, u)
$$

where $a_{i, j}(t, x, u)$ denotes the generic term of the symmetric matrix $\sigma \sigma^{*}(t, x, u)$. Let $U$ denote the class of admissible controls, that is, $\left(\mathscr{F}_{t}\right)_{t}$-adapted processes with values in the action space $\mathbb{A}$. This class is nonempty since it contains constant controls.

The cost function to be minimized over such controls is

$$
J(u)=E\left[\int_{0}^{1} h\left(t, x_{t}, u_{t}\right) d t+g\left(x_{1}\right)\right],
$$

where $h$ and $g$ are assumed to be real-valued, continuous, and bounded, respectively, on $\mathbb{R}_{+} \times \mathbb{R}^{d} \times \mathbb{A}$ and on $\mathbb{R}^{d}$.

We now introduce the notion of strict control to (2.1).

Definition 2.1. A strict control is the term $\alpha=\left(\Omega, \mathscr{F}_{t}, \mathscr{F}_{t}, P, u_{t}, x_{t}, x\right)$ such that

(1) $x \in \mathbb{R}^{d}$ is the initial data;

(2) $(\Omega, \mathscr{F}, P)$ is a probability space equipped with a filtration $\left(\mathscr{F}_{t}\right)_{t \geq 0}$ satisfying the usual conditions;

(3) $u_{t}$ is an $A$-valued process, progressively measurable with respect to $\left(\mathscr{F}_{t}\right)$;

(4) $\left(x_{t}\right)$ is $\mathbb{R}^{d}$-valued, $\mathscr{F}_{t}$-adapted, with continuous paths, such that

$$
f\left(x_{t}\right)-f(x)-\int_{0}^{t} L f\left(s, x_{s}, u_{s}\right) d s \text { is a } P \text {-martingale, }
$$

for each $f \in C_{b}^{2}$, for each $t>0$, where $L$ is the infinitesimal generator of the diffusion $\left(x_{t}\right)$.

In fact, when the control $u_{t}$ is constant, the conditions imposed above on the drift term and diffusion coefficient ensure that our martingale problem admits at least one solution, which implies weak existence of solutions of (2.1) (see [11]). The associated controls are called weak controls because of the possible change of the probability space and the Brownian motion with $u_{t}$. When pathwise uniqueness holds for the controlled equation it is shown in El Karoui et al. [5] that the weak and strong control problems are equivalent in the sense that they have the same value functions.

2.2. The relaxed control problem. The strict control problem as defined in Section 2.1 may fail to have an optimal solution, as shown in the following simple example, taken 
from deterministic control. See Fleming [7] and Yong and Zhou [25] for other examples from stochastic control. The problem is to minimize the following cost function:

$$
J(u)=\int_{0}^{T} x^{u}(t)^{2} d t
$$

over the set $U_{a d}$ of open loop controls, that is, measurable functions $u:[0, T] \rightarrow\{-1,1\}$.

Let $x^{u}(t)$ denote the solution of

$$
d x_{t}^{u}=u d t, \quad x(0)=0
$$

We have $\inf _{u \in \mathcal{U}} J(u)=0$. Indeed consider the following sequence of controls:

$$
u_{n}(t)=(-1)^{k} \quad \text { if } \frac{k}{n} \leq t \leq \frac{k+1}{n}, \quad 0 \leq k \leq n-1 .
$$

Then clearly $\left|x^{u_{n}}(t)\right| \leq 1 / n$ and $\left|J\left(u_{n}\right)\right| \leq T / n^{2}$ which implies that $\inf _{u \in \mathscr{U}} J(u)=0$. There is however no control $u$ such that $J(u)=0$. If this would have been the case, then for every $t, x^{u}(t)=0$. This in turn would imply that $u_{t}=0$, which is impossible. The problem is that the sequence $\left(u_{n}\right)$ has no limit in the space of strict controls. This limit, if it exists, will be the natural candidate for optimality. If we identify $u_{n}(t)$ with the Dirac measure $\delta_{u_{n}(t)}(d a)$ and set $q_{n}(d t, d u)=\delta_{u_{n}(t)}(d u) d t$, we get a measure on $[0,1] \times \mathbb{A}$. Then $\left(q_{n}(d t, d u)\right)_{n}$ converges weakly to $(1 / 2) d t \cdot\left[\delta_{-1}+\delta_{1}\right](d a)$. This suggests that the set $U$ of strict controls is too narrow and should be embedded into a wider class with a richer topological structure for which the control problem becomes solvable. The idea of relaxed control is to replace the $\mathbb{A}$-valued process $\left(u_{t}\right)$ with $\mathbb{P}(\mathbb{A})$-valued process $\left(\mu_{t}\right)$, where $\mathbb{P}(\mathbb{A})$ is the space of probability measures equipped with the topology of weak convergence.

In this section, we introduce relaxed controls of our systems of SDE as solutions of a martingale problem for a diffusion process whose infinitesimal generator is integarted against the random measures defined over the action space of all controls. Let $\mathbb{V}$ be the set of Radon measures on $[0,1] \times \mathbb{A}$ whose projections on $[0,1]$ coincide with the Lebesgue measure $d t$. Equipped with the topology of stable convergence of measures, $\mathbb{V}$ is a compact metrizable space (see Jacod and Mémin [12]). Stable convergence is required for bounded measurable functions $h(t, a)$ such that for each fixed $t \in[0,1], h(t, \cdot)$ is continuous.

Definition 2.2. A relaxed control is the term $\mu=\left(\Omega, \mathscr{F}_{,}, \mathscr{F}_{t}, P, B_{t}, \mu_{t}, x_{t}, x\right)$ such that

(1) $\left(\Omega, \mathscr{F}_{,}, \mathscr{F}_{t}, P\right)$ is a filtered probability space satisfying the usual conditions;

(2) $\left(\mu_{t}\right)_{t}$ is a $\mathbb{P}(\mathbb{A})$-valued process, progressively measurable with respect to $\left(\mathscr{F}_{t}\right)$ and such that for each $t, 1_{(0, t]} \cdot \mu$ is $F_{t}$-measurable;

(3) $\left(x_{t}\right)_{t}$ is $\mathbb{R}^{d}$-valued, $F_{t}$-adapted with continuous paths such that $x(0)=x$ and

$$
f\left(x_{t}\right)-f(x)-\int_{0}^{t} \int_{A} L f\left(s, x_{s}, a\right) \mu_{s}(\omega, d a) d s
$$

is a $P$-martingale, for each $f \in C_{b}^{2}\left(R^{d}, R\right)$.

We denote by $\mathscr{R}$ the collection of all relaxed controls. 
By a slight abuse of notation, we will often denote a relaxed control by $\mu$ instead of specifying all the components.

The cost function associated to a relaxed control $\mu$ is defined as

$$
J(\mu)=E\left[\int_{0}^{1} \int_{A} h\left(t, X_{t}, a\right) \mu_{t}(d a) d t+g\left(X_{1}\right)\right] .
$$

The set $U$ of strict controls is embedded into the set $\mathscr{R}$ of relaxed controls by the mapping

$$
\Psi: u \in U \longmapsto \Psi(u)(d t, d a)=d t \delta_{u(t)}(d a) \in \mathscr{R},
$$

where $\delta_{u}$ is the Dirac measure at a single point $u$. In fact the next lemma, known as the chattering lemma, tells us that any relaxed control is a weak limit of a sequence of strict controls. This lemma was first proved for deterministic measures in [8] and extended to random measures in $[6,7]$.

Lemмa 2.3 (chattering lemma). Let $\left(\mu_{t}\right)$ be a predictable process with values in the space of probability measures on $\mathbb{A}$. Then there exists a sequence of predictable processes $\left(u^{n}(t)\right)$ with values in $\mathbb{A}$ such that the sequence of random measures $\left(\delta_{u_{t}^{n}}(d a) d t\right)$ converges weakly to $\mu_{t}(d a) d t, P$-a.s.

In the next example, through considering the action space $\mathbb{A}$ to be a finite set of points, hence reducing the problem to controlling a finite-dimensional diffusion process, we will identify the appropriate class of martingale measures that drives the stochastic representation of the coordinate process associated with the solution to the martingale problem (2.8).

Example 2.4. Let the action space be the finite set $\mathbb{A}=\left\{a_{1}, a_{2}, \ldots, a_{n}\right\}$. Then every relaxed control $d t \mu_{t}(d a)$ will be a convex combination of the Dirac measures $d t \mu_{t}(d a)=$ $\sum_{i=1}^{n} \alpha_{t}^{i} d t \delta_{a_{i}}(d a)$, where for each $i, \alpha_{t}^{i}$ is a real-valued process such that $0 \leq \alpha_{t}^{i} \leq 1$ and $\sum_{i=1}^{n} \alpha_{t}^{i}=1$. It is not difficult to show that the solution of the (relaxed) martingale problem (2.8) is the law of the solution of the following SDE:

$$
d x_{t}=\sum_{i=1}^{n} b\left(t, x_{t}, a_{i}\right) \alpha_{t}^{i} d t+\sum_{i=1}^{n} \sigma\left(s, x_{s}, a_{i}\right)\left(\alpha_{t}^{i}\right)^{1 / 2} d B_{s}^{i}, \quad x_{0}=x,
$$

where the $B^{i}$ s are $d$-dimensional Brownian motions on an extension of the initial probability space. The process $M$ defined by

$$
M(A \times[0, t])=\sum_{i=1}^{n} \int_{0}^{t}\left(\alpha_{s}^{i}\right)^{1 / 2} 1_{\left\{a_{i} \in A\right\}} d B_{s}^{i}
$$

is in fact a strongly orthogonal continuous martingale measure (cf. Walsh [24], El-Karoui and Méléard [4]) with intensity $\mu_{t}(d a) d t=\sum \alpha_{t}^{i} \delta_{a_{i}}(d a) d t$. Thus, the SDE in (2.11) can be expressed in terms of $M$ and $\mu$ as follows:

$$
d x_{t}=\int_{\mathbb{A}} b\left(t, x_{t}, a\right) \mu_{t}(d a) d t+\int_{\mathbb{A}} \sigma\left(t, x_{t}, a\right) M(d a, d t) .
$$


The following theorem due to El Karoui and Méléard [4] shows in fact a general representation result for solutions of the martingale problem (2.8) in terms of strongly orthogonal continuous martingale measures whose intensities are our relaxed controls.

Theorem 2.5 [4]. (1) Let $P$ be the solution of the martingale problem (2.8). Then $P$ is the law of a d-dimensional adapted and continuous process $X$ defined on an extension of the space $\left(\Omega, \mathscr{F}_{t}, \mathscr{F}_{t}\right)$ and solution of the following SDE starting at $x$ :

$$
d X_{t}^{i}=\int_{\mathbb{A}} b_{i}\left(t, X_{t}, a\right) \mu_{t}(d a) d t+\sum_{k=1}^{d} \int_{\mathbb{A}} \sigma_{i, k}\left(t, X_{t}, a\right) M^{k}(d a, d t),
$$

where $M=\left(M^{k}\right)_{k=1}^{d}$ is a family of d-strongly orthogonal continuous martingale measures with intensity $\mu_{t}(d a) d t$.

(2) If the coefficients $b$ and $\sigma$ are Lipschitz in $x$, uniformly in $t$ and $a$, the SDE (2.14) has a unique pathwise solution.

Using the chattering lemma, we get the following result due to Méléard [14] on approximating continuous orthogonal martingale measures with given intensity with a sequence of stochastic integrals with respect to a single Brownian motion. See also $[15,16]$ for applications of martingale measures in infinite systems of interacting particles and branching processes.

Proposition 2.6 [14]. Let M be a continuous orthogonal martingale measure with intensity $\mu_{t}(d a) d t$ on $\mathbb{A} \times[0,1]$. Then there exist a sequence of predictable $\mathbb{A}$-valued processes $\left(u^{n}(t)\right)$ and a Brownian motion $B$ defined on an extension of $(\Omega, \mathscr{F}, P)$ such that for all $t \in[0, T]$ and $\varphi$ continuous bounded functions from $\mathbb{A}$ to $\mathbb{R}$,

$$
\lim _{n \rightarrow+\infty} E\left[\left(M_{t}(\varphi)-\int_{0}^{t} \varphi\left(u^{n}(s) d B_{s}\right)\right)^{2}\right]=0 .
$$

\section{Approximation and existence results of relaxed controls}

In order for the relaxed control problem to be truly an extension of the original one, the infimum of the expected cost among relaxed controls must be equal to the infimum among strict controls. This result is based on the approximation of a relaxed control by a sequence of strict controls, given by Lemma 2.3.

The next theorem which is our main result in this section gives the stability of the controlled stochastic differential equations with respect to the control variable.

Let $\left(\mu_{t}\right)$ be a relaxed control. We know from Theorem 2.5 , that there exists a family of continuous strongly orthogonal martingale measures $M_{t}=\left(M_{t}^{k}\right)$ such that the state of the system satisfies the following SDE, starting at $X_{0}=x$ :

$$
d X_{t}=\int_{A} b\left(t, X_{t}, a\right) \mu_{t}(d a) d t+\int_{A} \sigma\left(t, x_{t}, a\right) M(d a, d t) .
$$

Moreover, thanks to Lemma 3.4 and Proposition 2.6, there exist a sequence $\left(u^{n}(t)\right)$ of strict controls and a Brownian motion $B$ defined on an extension of $(\Omega, \mathscr{F}, P)$ such that 
for each $t \in[0, T]$ and each continuous bounded function $\varphi$ from $\mathbb{A}$ to $\mathbb{R}$,

$$
\lim _{n \rightarrow+\infty} E\left[\left(M_{t}(\varphi)-\int_{0}^{t} \varphi\left(u^{n}(s)\right) d B_{s}\right)^{2}\right]=0 .
$$

Denote by $X_{t}^{n}$ the solution of

$$
\begin{gathered}
d X_{t}^{n}=b\left(t, X_{t}^{n}, u_{t}^{n}\right) d t+\sigma\left(t, X_{t}^{n}, u_{t}^{n}\right) d B_{t}, \\
X^{n}(0)=x,
\end{gathered}
$$

which can be written in relaxed form as

$$
\begin{gathered}
d X_{t}^{n}=\int_{A} b\left(t, X_{t}^{n}, a\right) \mu_{t}^{n}(d a) d t+\int_{A} \sigma\left(t, X_{t}^{n}, a\right) M^{n}(d a, d t), \\
X_{0}^{n}=x
\end{gathered}
$$

with respect to the martingale measure $M^{n}(t, A)=\int_{0}^{t} 1_{A}\left(u^{n}(s)\right) d B_{s}$ and $\mu_{t}^{n}(d a)=\delta_{u^{n}(t)}(d a)$. Theorem 3.1. Let $X_{t}$ and $X_{t}^{n}$ be the diffusions solutions of (3.1) and (3.4), respectively. If the pathwise uniqueness holds for (3.1), then

$$
\lim _{n \rightarrow \infty} E\left[\sup _{0 \leq t \leq 1}\left|X_{t}^{n}-X_{t}\right|^{2}\right]=0 .
$$

The proof of Theorem 3.1 will be given later.

Corollary 3.2. Let $J\left(u^{n}\right)$ and $J(\mu)$ be the expected costs corresponding, respectively, to $u^{n}$ and $\mu$, where $u^{n}$ and $\mu$ are defined as in the last theorem. Then there exists a subsequence $\left(u^{n_{k}}\right)$ of $\left(u^{n}\right)$ such that $J\left(u^{n_{k}}\right)$ converges to $J(\mu)$.

Proof of Corollary 3.2. From Theorem 3.1 it follows that the sequence $\left(X_{t}^{n}\right)$ converges to $X_{t}$ in probability, uniformly in $t$, then there exists a subsequence $\left(X_{t}^{n_{k}}\right)$ that converges to $X_{t}, P$-a.s., uniformly in $t$. We have

$$
\begin{aligned}
\left|J\left(u^{n_{k}}\right)-J(\mu)\right| \leq & E\left[\int_{0}^{1} \int_{\mathbb{A}}\left|h\left(t, X_{t}^{n_{k}}, a\right)-h\left(t, X_{t}, a\right)\right| \delta_{u_{t}^{n_{k}}}(d a) d t\right] \\
& +E\left[\left|\int_{0}^{1} \int_{\mathbb{A}} h\left(t, X_{t}, a\right) \delta_{u_{t}^{n_{k}}}(d a) d t-\int_{0}^{1} \int_{\mathbb{A}} h\left(t, X_{t}, a\right) \mu_{t}(d a) d t\right|\right] \\
& +E\left[\left|g\left(X_{1}^{n_{k}}\right)-g\left(X_{1}\right)\right|\right] .
\end{aligned}
$$

It follows from the continuity and boundness of the functions $h$ and $g$ with respect to $x$ that the first and third terms in the right-hand side converge to 0 . The second term in the right-hand side tends to 0 by the weak convergence of the sequence $\delta_{u^{n}}$ to $\mu$, the continuity and the boundness of $h$ in the variable $a$. We use the dominated convergence theorem to conclude.

To prove Theorem 3.1, we need some auxiliary results on the tightness of the processes in question. 
Lemma 3.3. The family of relaxed controls $\left(\left(\mu^{n}\right)_{n \geq 0}, \mu\right)$ is tight in $V$.

Proof. $[0,1] \times \mathbb{A}$ being compact, then by Prokhorov's theorem, the space $\mathbb{V}$ of probability measures on $[0,1] \times \mathbb{A}$ is also compact for the topology of weak convergence. The fact that $\mu^{n}, n \geq 0$ and $\mu$ being random variables with values in the compact set $\mathbb{V}$ yields that the family of distributions associated to $\left(\left(\mu^{n}\right)_{n \geq 0}, \mu\right)$ is tight.

Lemma 3.4. The family of martingale measures $\left(\left(M^{n}\right)_{n \geq 0}, M\right)$ is tight in the space $C_{S^{\prime}}=$ $C\left([0,1], S^{\prime}\right)$ of continuous functions from $[0,1]$ with values in $S^{\prime}$ the topological dual of the Schwartz space $S$ of rapidly decreasing functions.

Proof. The martingale measures $M^{n}, n \geq 0, M$ can be considered as random variables with values in $C_{S^{\prime}}=C\left([0,1], S^{\prime}\right)$ (see Mitoma [20]). By applying [20, Lemma 6.3], it is sufficient to show that for every $\varphi$ in $S$, the family $\left(M^{n}(\varphi), n \geq 0, M(\varphi)\right)$ is tight in $C\left([0,1], \mathbb{R}^{d}\right)$, where $M^{n}(\omega, t, \varphi)=\int_{\mathbb{A}} \varphi(a) M^{n}(\omega, t, d a)$ and $M(\omega, t, \varphi)=\int_{\mathbb{A}} \varphi(a) M(\omega, t, d a)$. Let $p>1$ and $s<t$. By the Burkholder-Davis-Gundy inequality, we have

$$
\begin{aligned}
E\left(\left|M_{t}^{n}(\varphi)-M_{s}^{n}(\varphi)\right|^{2 p}\right) & \leq C_{p} E\left[\left(\int_{s}^{t} \int_{\mathbb{A}}|\varphi(a)|^{2} \mu_{t}^{n}(d a) d t\right)^{p}\right] \\
& =C_{p} E\left[\left(\int_{s}^{t}\left|\varphi\left(u_{t}^{n}\right)\right|^{2} d t\right)^{p}\right] \\
& \leq C_{p} \sup _{a \in \mathbb{A}}|\varphi(a)|^{2 p}|t-s|^{p} \\
& \leq K_{p}|t-s|^{p},
\end{aligned}
$$

where $K_{p}$ is a constant depending only on $p$. That is the Kolmogorov condition is fulfilled (see Lemma A.2 in the appendix below). Hence the sequence $\left(M^{n}(\varphi)\right)$ is tight. The same arguments can be used to show that $E\left(\left|M_{t}(\varphi)-M_{s}(\varphi)\right|^{2 p}\right) \leq K_{p}|t-s|^{p}$, which yields the tightness of $M_{t}(\varphi)$.

Lemma 3.5. If $X_{t}$ and $X_{t}^{n}$ are the solutions of (5) and (6), respectively, then the family of processes $\left(X_{t}, X_{t}^{n}\right)$ is tight in $C=C\left([0,1], \mathbb{R}^{d}\right)$.

Proof. Let $p>1$ and $s<t$. Using the usual arguments from stochastic calculus and the boundness of the coefficients $b$ and $\sigma$, it is easy to show that

$$
E\left(\left|X_{t}^{n}-X_{s}^{n}\right|^{2 p}\right) \leq C_{p}|t-s|^{p}, \quad E\left(\left|X_{t}-X_{s}\right|^{2 p}\right) \leq C_{p}|t-s|^{p}
$$

which yields the tightness of $\left(X_{t}, X_{t}^{n}, n \geq 0\right)$.

Proof of Theorem 3.1. Let $\mu$ be a relaxed control. According to Lemma 2.3, there exists a sequence $\left(u^{n}\right) \subset \mathcal{U}$ such that $\mu^{n}=d t \delta_{u^{n}(t)}(d a)$ converges to $d t \mu(t, d a)$ in $\mathbb{V}, P$-a.s. Let $X_{t}^{n}$ and $X_{t}$ be the solutions of (3.4) and (3.1) associated with $\mu$ and $u^{n}$. Suppose that the conclusion of Theorem 3.1 is false. Then there exists $\beta>0$ such that

$$
\inf _{n} E\left[\left|X_{t}^{n}-X_{t}\right|^{2}\right] \geq \beta
$$


Seïd Bahlali et al. 9

According to Lemmas 3.3, 3.4, and 3.5, the family of processes

$$
\gamma^{n}=\left(\mu^{n}, \mu, M^{n}, M, X^{n}, X\right)
$$

is tight in the space

$$
\Gamma=(\mathbb{V} \times \mathbb{V}) \times\left(C_{S^{\prime}} \times C_{S^{\prime}}\right) \times(C \times C) .
$$

By the Skorokhod selection theorem (Lemma A.1 in the appendix below), there exist a probability space $(\hat{\Omega}, \widehat{\mathscr{F}}, \hat{P})$ and a sequence $\hat{\gamma}^{n}=\left(\hat{\mu}^{n}, \widehat{v}^{n}, \hat{X}^{n}, \hat{Y}^{n}, \widehat{M}^{n}, \hat{N}^{n}\right)$ defined on it such that

(i) for each $n \in \mathbb{N}$, the laws of $\gamma^{n}$ and $\hat{\gamma}^{n}$ coincide,

(ii) there exists a subsequence $\left(\hat{\gamma}^{n_{k}}\right)$ of $\left(\hat{\gamma}^{n}\right)$ still denoted by $\left(\hat{\gamma}^{n}\right)$ which converges to $\hat{\gamma}, \hat{P}$-a.s. on the space $\Gamma$, where $\hat{\gamma}=(\hat{\mu}, \hat{v}, \hat{X}, \hat{Y}, \widehat{M}, \hat{N})$.

By the uniform integrability, it holds that

$$
\beta \leq \liminf _{n} E\left[\sup _{t \leq 1}\left|X_{t}^{n}-X_{t}\right|^{2}\right]=\liminf _{n} \hat{E}\left[\sup _{t \leq 1}\left|\hat{X}_{t}^{n}-\hat{Y}_{t}^{n}\right|^{2}\right]=\hat{E}\left[\sup _{t \leq 1}\left|\hat{X}_{t}-\hat{Y}_{t}\right|^{2}\right],
$$

where $\hat{E}$ is the expectation with respect to $\hat{P}$. According to property (i), we see that $\hat{X}_{t}^{n}$ and $\hat{Y}_{t}^{n}$ satisfy the following equations:

$$
\begin{array}{ll}
d \hat{X}_{t}^{n}=\int_{A} b\left(t, \hat{X}_{t}^{n}, a\right) \hat{\mu}^{n}(t, d a) d t+\int_{A} \sigma\left(t, \hat{X}_{t}^{n}, a\right) \widehat{M}^{n}(d a, d t), & \hat{X}_{0}^{n}=x, \\
d \hat{Y}_{t}^{n}=\int_{A} b\left(t, \hat{Y}_{t}^{n}, a\right) \hat{v}^{n}(t, d a) d t+\int_{A} \sigma\left(t, \hat{X}_{t}^{n}, a\right) \widehat{N}^{n}(d a, d t), & \hat{Y}_{0}^{n}=x .
\end{array}
$$

Since $b$ and $\sigma$ are continuous in $(x, a)$, then using the fact that $\left(\hat{\gamma}^{n}\right)$ converges to $\hat{\gamma}, \hat{P}$ a.s., it holds that $\int_{0}^{t} \int_{A} b\left(t, \hat{X}_{t}^{n}, a\right) \widehat{\mu}^{n}(t, d a) d t$ converges in probability to $\int_{0}^{t} \int_{A} b\left(t, \hat{X}_{t}, a\right) \hat{\mu}(t$, $d a) d t$, and $\int_{A} \sigma\left(t, \hat{X}_{t}^{n}, a\right) \widehat{M}^{n}(d a, d t)$ converges in probability to $\int_{A} \sigma\left(t, \hat{X}_{t}, a\right) \widehat{M}(d a, d t)$.

The same claim holds for the second equation in (3.13). Hence, $\left(\hat{X}_{t}^{n}\right)$ and $\left(\hat{Y}_{t}^{n}\right)$ converge, respectively, to $\hat{X}_{t}$ and $\hat{Y}_{t}$ which satisfy

$$
\begin{array}{ll}
d \hat{X}_{t}=\int_{A} b\left(t, \hat{X}_{t}, a\right) \hat{\mu}(t, d a) d t+\int_{A} \sigma\left(t, \hat{X}_{t}, a\right) \widehat{M}(d a, d t), & \hat{X}_{0}=x \\
d \hat{Y}_{t}=\int_{A} b\left(t, \hat{Y}_{t}, a\right) \hat{v}(t, d a) d t+\int_{A} \sigma\left(t, \hat{Y}_{t}, a\right) \hat{N}(d a, d t), & \hat{Y}_{0}=x .
\end{array}
$$

The rest of the proof consists in showing that $\widehat{\mu}=\widehat{v}, \widehat{P}$-a.s., and $\widehat{M}(d a, d t)=\hat{N}(d a, d t)$, $\hat{P}$-a.s. By Lemma $2.3, \mu^{n} \rightarrow \mu$ in $\mathbb{V}, P$-a.s, it follows that the sequence $\left(\mu^{n}, \mu\right)$ converges to $(\mu, \mu)$ in $\mathbb{V}^{2}$. Moreover,

$$
\operatorname{law}\left(\mu^{n}, \mu\right)=\operatorname{law}\left(\hat{\mu}^{n}, \hat{v}^{n}\right)
$$

and as $n \rightarrow \infty$,

$$
\left(\hat{\mu}^{n}, \hat{v}^{n}\right) \longrightarrow(\hat{\mu}, \hat{v}), \quad \hat{P} \text {-a.s. in } \mathbb{V}^{2} \text {. }
$$


Therefore law $(\hat{\mu}, \hat{v})=\operatorname{law}(\mu, \mu)$ which is supported by the diagonal of $\mathbb{V}^{2}$. Hence $\hat{\mu}=\hat{v}, \hat{P}$ a.s.

The same arguments may be applied to show that $\widehat{M}(d a, d t)=\widehat{N}(d a, d t), \widehat{P}$-a.s. It follows that $\hat{X}$ and $\hat{Y}$ are solutions of the same stochastic differential equation with the same martingale measure $\widehat{M}$ and the same relaxed control $\hat{\mu}$. Hence by the pathwise uniqueness property we have $\hat{X}=\hat{Y}, \hat{P}$-a.s., which contradicts (3.9).

Using Skorokhod selection theorem, we show in the next proposition that an optimal solution for the relaxed control problem exists. Note that another proof based on martingale problems of the type (2.8) is given in El-Karoui et al. [5].

Proposition 3.6. Suppose that the coefficients $b, \sigma, h$, and $g$ are bounded, measurable, and continuous in $(x, a)$. Then the relaxed control problem admits an optimal solution.

Proof. Let $\beta=\inf \{J(\mu) ; \mu \in \mathscr{R}\}$, where

$$
J(\mu)=E\left[\int_{0}^{1} \int_{A} h\left(t, X_{t}, a\right) \mu_{t}(d a) d t+g\left(X_{T}\right)\right] .
$$

Let $\left(\mu^{n}, X^{n}\right)_{n \geq 0}$ be a minimizing sequence for the cost function $J(\mu)$, that is, $\lim _{n \rightarrow+\infty} J\left(\mu^{n}\right)$ $=\beta$, where $X^{n}$ is the solution of

$$
d X_{t}^{n}=\int_{A} b\left(t, X_{t}^{n}, a\right) \mu_{t}^{n}(d a) d t+\int_{A} \sigma\left(t, X_{t}^{n}, a\right) M^{n}(d a, d t), \quad X_{0}^{n}=x .
$$

Using the same arguments as in the proof of Theorem 3.1, it holds that $\gamma^{n}=\left(\mu^{n}, M^{n}, X^{n}\right)$ is tight in the space $\Gamma=\mathbb{V} \times C_{S^{\prime}} \times C$. Moreover, using the Skorokhod selection theorem (Lemma A.1 in the appendix), there exist a probability space $(\hat{\Omega}, \hat{\mathscr{F}}, \hat{P})$ and a sequence $\hat{\gamma}^{n}=\left(\widehat{\mu}^{n}, \widehat{M}^{n}, \hat{X}^{n}\right)$ defined on it such that

(i) for each $n \in \mathbb{N}$, the laws of $\gamma^{n}$ and $\hat{\gamma}^{n}$ coincide;

(ii) there exists a subsequence $\left(\hat{\gamma}^{n_{k}}\right)$ of $\left(\hat{\gamma}^{n}\right)$ still denoted by $\left(\hat{\gamma}^{n}\right)$ which converges to $\hat{\gamma}, \hat{P}$-a.s., on the space $\Gamma$, where $\hat{\gamma}=(\hat{\mu}, \widehat{M}, \hat{X})$.

According to property (i), we see that $\hat{X}_{t}^{n}$ satisfies the following equation:

$$
d \widehat{X}_{t}^{n}=\int_{A} b\left(t, \hat{X}_{t}^{n}, a\right) \hat{\mu}^{n}(t, d a) d t+\int_{A} \sigma\left(t, \hat{X}_{t}^{n}, a\right) \widehat{M}^{n}(d a, d t), \quad \hat{X}_{0}^{n}=x
$$

Since $b$ and $\sigma$ are continous in $(x, a)$, then using the fact that $\left(\hat{\gamma}^{n}\right)$ converges to $\hat{\gamma}, \widehat{P}$-a.s., it holds that $\int_{0}^{t} \int_{A} b\left(t, \hat{X}_{t}^{n}, a\right) \hat{\mu}^{n}(t, d a) d t$ converges in probability to $\int_{0}^{t} \int_{A} b\left(t, \hat{X}_{t}, a\right) \hat{\mu}(t, d a) d t$, and $\int_{A} \sigma\left(t, \widehat{X}_{t}^{n}, a\right) \widehat{M}^{n}(d a, d t)$ converges in probability to $\int_{A} \sigma\left(t, \hat{X}_{t}, a\right) \widehat{M}(d a, d t)$.

Hence, $\left(\hat{X}_{t}^{n}\right)$ and $\left(\hat{Y}_{t}^{n}\right)$ converge, respectively, to $\hat{X}_{t}$ and $\hat{Y}_{t}$ which satisfy

$$
d \hat{X}_{t}=\int_{A} b\left(t, \hat{X}_{t}, a\right) \hat{\mu}(t, d a) d t+\int_{A} \sigma\left(t, \hat{X}_{t}, a\right) \widehat{M}(d a, d t), \quad \hat{X}_{0}=x
$$

The instantaneous cost $h$ and the final cost $g$ being continuous and bounded in $(x, a)$, we proceed as in Corollary 3.2, to conclude that $\beta=\lim _{n \rightarrow+\infty} J\left(\mu^{n}\right)=J(\hat{\mu})$. Hence $\hat{\mu}$ is an optimal control. 


\section{Maximum principle for relaxed control problems}

4.1. Assumptions and preliminaries. In this section we establish optimality necessary conditions for relaxed control problems, where the system is described by a SDE driven by a martingale measure of the form (3.1) and the admissible controls are measure-valued processes. The proof is again based on the chattering lemma, where, using Ekeland's variational principle, we derive necessary conditions of near optimality for the approximating sequence of strict controls. We obtain the maximum principle for our relaxed control problem by using stability properties of the corresponding state equations and adjoint processes.

Recall the controlled SDE:

$$
d x_{t}=\int_{A} b\left(t, x_{t}, a\right) \mu_{t}(d a) d t+\int_{A} \sigma\left(t, x_{t}, a\right) M(d a, d t), \quad x_{0}=x
$$

where $M(d a, d t)$ is an orthogonal martingale mesure whose intensity is the relaxed control $\mu_{t}(d a) d t$. The corresponding cost is given by

$$
J(\mu)=E\left[g\left(x_{1}\right)+\int_{0}^{1} \int_{A} h\left(t, x_{t}, a\right) \mu_{t}(d a)\right]
$$

We assume that the coefficients of the controlled equation satisfy the following hypothesis.

$(\mathrm{H} 1) \quad b: \mathbb{R}_{+} \times \mathbb{R}^{d} \times \mathbb{A} \rightarrow \mathbb{R}^{d}, \sigma: \mathbb{R}_{+} \times \mathbb{R}^{d} \times \mathbb{A} \rightarrow \mathbb{R}^{d} \otimes \mathbb{R}^{k}$, and $h: \mathbb{R}_{+} \times \mathbb{R}^{d} \times \mathbb{A} \rightarrow \mathbb{R}$ are bounded measurable in $(t, x, a)$ and twice continuously differentiable functions in $x$ for each $(t, a)$, and there exists a constant $C>0$ such that

$$
|f(t, x, a)-f(t, y, a)|+\left|f_{x}(t, x, a)-f_{x}(t, y, a)\right| \leq C|x-y|,
$$

where $f$ stands for one of the functions $b, \sigma, h$.

$b, \sigma, h$ and their first and second derivatives are continuous in the control variable $a$.

$g: \mathbb{R}^{d} \rightarrow \mathbb{R}$ is bounded and twice continuously differentiable such that

$$
|g(y)-g(y)|+\left|g_{x}(y)-g_{x}(y)\right| \leq C|x-y| \text {. }
$$

Under the assumptions above, the controlled equation admits a unique strong solution such that for every $p \geq 1, E\left[\sup _{0 \leq t \leq T}\left|x_{t}\right|^{p}\right]<M(p)$.

We know by Proposition 3.6 that an optimal relaxed control denoted by $\mu$ exists. We seek for necessary conditions satisfied by this control in a form similar to the Pontryagin maximum principle.

The next lemma is an approximation result which we prove directly without using Skorokhod's selection theorem, the coefficients being smooth in the state variable. 
Lemma 4.1. Let $\mu$ be a relaxed optimal control and $X$ the corresponding optimal trajectory. Then there exists a sequence $\left(u^{n}\right)_{n} \subset \mathcal{U}$ of strict controls such that

$$
\begin{gathered}
\lim _{n \rightarrow \infty} E\left[\sup _{0 \leq t \leq T}\left|X_{t}-X_{t}^{n}\right|^{2}\right]=0, \\
\lim _{n \rightarrow \infty} J\left(\mu^{n}\right)=J(\mu)
\end{gathered}
$$

where $\mu^{n}=d t \delta_{u_{t}^{n}}(d a)$ and $X^{n}$ denotes the solution of equation associated with $\mu^{n}$.

Proof. (i) The sequence $\left(u^{n}\right)$ is given by the chattering lemma (Lemma 2.3). Let $x$ and $x^{n}$ be the trajectories associated, respectively, with $\mu$ and $\mu^{n}$, and $t \in[0, T]$,

$$
\begin{aligned}
E\left[\left|x_{t}-x_{t}^{n}\right|^{2}\right]=E\left[\mid \int_{0}^{t} \int_{A} b\left(s, x_{s}^{n}, a\right) \delta_{u_{s}^{n}}(d a) d s+\int_{0}^{t} \sigma\left(s, x_{s}^{n}, u_{s}^{n}\right) d B_{s} .\right. \\
\left.-\int_{0}^{t} \int_{A} b\left(s, x_{s}, a\right) \mu_{s}(d a) d s+\left.\int_{0}^{t} \int_{A} \sigma\left(s, x_{s}, a\right) M(d s, d a)\right|^{2}\right] \\
\leq C_{1}\left\{E\left[\left|\int_{0}^{t} \int_{A} b\left(s, x_{s}^{n}, a\right) \delta_{u_{s}^{n}}(d a) d s-\int_{0}^{t} \int_{A} b\left(s, x_{s}, a\right) \mu_{s}(d a) d s\right|^{2}\right]\right. \\
\left.+E\left[\left|\int_{0}^{t} \sigma\left(s, x_{s}^{n}, u_{s}^{n}\right) d B_{s}-\int_{0}^{t} \int_{A} \sigma\left(s, x_{s}, a\right) M(d s, d a)\right|^{2}\right]\right\} \\
\leq C_{2}\left\{E\left[\left|\int_{0}^{t} b\left(s, x_{s}^{n}, u_{s}^{n}\right) d s-\int_{0}^{t} b\left(s, x_{s}, u_{s}^{n}\right) d s\right|^{2}\right]\right. \\
+E\left[\left|\int_{0}^{t} \int_{A} b\left(s, x_{s}, a\right) \delta_{u_{s}^{n}}(d a) d s-\int_{0}^{t} \int_{A} b\left(s, x_{s}, a\right) \mu_{s}(d a) d s\right|^{2}\right] \\
+E\left[\left|\int_{0}^{t} \sigma\left(s, x_{s}^{n}, u_{s}^{n}\right) d B_{s}-\int_{0}^{t} \int_{A} \sigma\left(s, x_{s}, u_{s}^{n}\right) d B_{s}\right|^{2}\right] \\
\left.+E\left[\left|\int_{0}^{t} \sigma\left(s, x_{s}, u_{s}^{n}\right) d B_{s}-\int_{0}^{t} \int_{A} \sigma\left(s, x_{s}, a\right) M(d s, d a)\right|^{2}\right]\right\} \\
=C_{2}\left\{I_{1}+I_{2}+I_{3}+I_{4}\right\} .
\end{aligned}
$$

Since the coefficients $b$ and $\sigma$ are Lipschitz continuous in the state variable $x$, then

$$
I_{1}+I_{3} \leq C_{3} E\left[\int_{0}^{T}\left|x_{s}^{n}-x_{s}\right|^{2} d s\right]
$$

Since $d t \delta_{u_{t}^{n}}(d a) \underset{n \rightarrow \infty}{\longrightarrow} d t \mu_{t}(d a)$ in $\mathbb{V}, P$-a.s, $b$ is bounded and continuous in the control variable $a$, therefore using the dominated convergence theorem, $I_{3}$ converges to 0 as $n$ tends to $+\infty$. 
We use Proposition 2.6 to prove that $I_{4}$ converges to 0 as $n$ tends to $+\infty$. Using Gronwall's lemma, we conclude that

$$
\lim _{n \rightarrow \infty} E\left[\left|x_{t}-x_{t}^{n}\right|^{2}\right]=0
$$

Applying Burkholder-Davis-Gundy inequality for the martingale part allows us to obtain a uniform convergence in $t$ :

$$
\lim _{n \rightarrow \infty} E\left[\sup _{0 \leq t \leq T}\left|x_{t}-x_{t}^{n}\right|^{2}\right]=0
$$

(ii)

$$
\begin{aligned}
\left|J(\mu)-J\left(\mu^{n}\right)\right| \leq & \left\{E\left[\left|\int_{0}^{t} h\left(s, x_{s}^{n}, u_{s}^{n}\right) d s-\int_{0}^{t} h\left(s, x_{s}, u_{s}^{n}\right) d s\right|^{2}\right]\right. \\
& +E\left[\left|\int_{0}^{t} \int_{A} h\left(s, x_{s}, a\right) \delta_{u_{s}^{n}}(d a) d s-\int_{0}^{t} \int_{A} h\left(s, x_{s}, a\right) \mu_{s}(d a) d s\right|^{2}\right] \\
& \left.+E\left[\left|g\left(X_{T}\right)-g\left(X_{T}^{n}\right)\right|\right]\right\} .
\end{aligned}
$$

The first and the third terms in the right-hand side converge to 0 because $h$ and $g$ are Lipschitz continuous in $x$ and the fact that

$$
\lim _{n \rightarrow \infty} E\left[\sup _{0 \leq t \leq T}\left|x_{t}-x_{t}^{n}\right|^{2}\right]=0
$$

$h$ is bounded and continuous in $a$; hence an application of the dominated convergence theorem allows us to conclude that the second term in the right-hand side tends to 0 .

4.2. Necessary conditions for near optimality. According to the optimality of $\mu$, there exists a sequence $\left(\epsilon_{n}\right)$ of positive real numbers with $\lim _{n \rightarrow+\infty} \epsilon_{n}=0$ such that

$$
J\left(u^{n}\right)=J\left(\mu^{n}\right) \leq \inf \{J(\mu) ; \mu \in \mathscr{R}\}+\epsilon_{n},
$$

where $\mu^{n}=d t \delta_{u_{t}^{n}}(d a)$.

In this section, we give necessary conditions for near optimality satisfied by the minimizing sequence $\left(u^{n}\right)$.

4.2.1. Stability of the state equation and adjoint processes. To derive necessary conditions for near optimality, we use Ekeland's variational principle (Lemma 4.2), along with an appropriate choice of a metric on the space $U$ of admissible controls. 
Lemma 4.2 (Ekeland). Let $(V, d)$ be a complete metric space and $F: V \rightarrow \mathbb{R} \cup\{+\infty\}$ be lower semicontinuous and bounded from below. Given $\epsilon>0$, suppose $u^{\epsilon} \in V$ satisfies $F\left(u^{\epsilon}\right)$ $\leq \inf \{F(v) ; v \in V\}+\epsilon$. Then for any $\lambda>0$, there exists $v \in V$ such that

(i) $F(v) \leq F\left(u^{\epsilon}\right)$;

(ii) $d\left(u^{\epsilon}, v\right) \leq \lambda$;

(iii) for all $w \neq v ; F(v)<F(w)+\varepsilon / \lambda \cdot d(w, v)$.

We denote by $d$ the metric on the space $\mathcal{U}$ defined by

$$
d(u, v)=P \otimes d t\{(\omega, t) \in \Omega \times[0, T] ; u(\omega, t) \neq v(\omega, t)\},
$$

where $P \otimes d t$ is the product measure of $P$ and the Lebesgue measure.

LEMMA 4.3. (i) $(\mathcal{U}, d)$ is a complete metric space.

(ii) For any $p \geq 1$, there exists $M>0$ such that for any $u, v \in \mathcal{U}$ the following estimate holds:

$$
E\left[\sup _{0 \leq t \leq T}\left|x_{t}^{u}-x_{t}^{v}\right|^{2 p}\right] \leq M \cdot(d(u, v))^{1 / 2}
$$

where $x_{t}^{u}$ and $x_{t}^{v}$ are the solutions of (2.1) corresponding to $u$ and $v$.

(iii) The cost functional $J:(U, d) \rightarrow \mathbb{R}$ is continuous. More precisely if $u$ and $v$ are two elements in $U$, then

$$
|J(u)-J(v)| \leq C \cdot(d(u, v))^{1 / 2} .
$$

See $[17,26]$ for the detailed proof.

For any strict control $u \in \mathcal{U}$, we denote $\left(p_{1}, Q_{1}\right)$ and $\left(p_{2}, Q_{2}\right)$ the first- and secondorder adjoint variables satisfying the following backward SDE are called sometimes adjoint equations:

$$
\begin{aligned}
& d p_{1}(t)=-[ b_{x}^{*}(t, x(t), u(t)) p_{1}(t)+\sigma_{x}^{*}(t, x(t), u(t)) Q_{1}(t) \\
&\left.+h_{x}(t, x(t), u(t))\right] d t+Q_{1}(t) d B_{t}, \\
& p_{1}(T)=g_{x}(x(T)), \\
&-d p_{2}(t)=-[ b_{x}^{*}(t, x(t), u(t)) p_{2}(t)+p_{2}(t) b_{x}(t, x(t), u(t)) \\
&+ \sigma_{x}^{*}(t, x(t), u(t)) p_{2}(t) \sigma_{x}(t, x(t), u(t)) \\
&+ \sigma_{x}^{*}(t, x(t), u(t)) Q_{2}(t) \\
&+\left.Q_{2}(t) \sigma_{x}\left(t, x(t), u(t)-H_{x x}\left(x(t), u(t), p_{1}(t), Q_{1}(t)\right)\right)\right] d t \\
&+Q_{2}(t) d B_{t}, \\
& p_{2}(T)=g_{x x}(x(T)),
\end{aligned}
$$

where $x(t)$ is the trajectory associated with $u$. 
The derivatives of the data being bounded, the generators of these backward SDEs are globally Lipschitz and have linear growth. Then by the result of Pardoux and Peng [21], there exist unique $F_{t}$-adapted pairs $\left(p_{1}, Q_{1}\right)$ and $\left(p_{2}, Q_{2}\right)$ with values, respectively, in $\mathbb{R}^{d} \times \mathbb{R}^{d \times d}$ and $\mathbb{R}^{d \times d} \times\left(\mathbb{R}^{d \times d}\right)^{d}$ which solve (4.16) such that

$$
\begin{aligned}
& E\left[\sup _{0 \leq t \leq T}\left|p_{1}(t)\right|^{2}+\int_{0}^{T}\left|Q_{1}(t)\right|^{2} d t\right]<+\infty, \\
& E\left[\sup _{0 \leq t \leq T}\left|p_{2}(t)\right|^{2}+\int_{0}^{T}\left|Q_{2}(t)\right|^{2} d t\right]<+\infty .
\end{aligned}
$$

Lemma 4.4. For any $0<\alpha<1$ and $1<p<2$ satisfying $(1+\alpha)<2$, there exists a constant $C_{1}=C_{1}(\alpha, p)>0$ such that for any strict controls $u$ and $u^{\prime}$ along with the corresponding trajectories $x$ and $x^{\prime}$ and the solutions $\left(p_{1}^{\prime}, Q_{1}^{\prime}, p_{2}^{\prime}, Q_{2}^{\prime}\right)$ and $\left(p_{1}^{\prime}, Q_{1}^{\prime}, p_{2}^{\prime}, Q_{2}^{\prime}\right)$ of the backward $\operatorname{SDE}(\cdot)$ and $(\cdot)$, the following estimates hold:

$$
\begin{aligned}
& E\left[\int_{0}^{T}\left(\left|p_{1}(t)-p_{1}^{\prime}(t)\right|^{2}+\left|Q_{1}(t)-Q_{1}^{\prime}(t)\right|^{2}\right) d t\right] \leq C_{1} d\left(u, u^{\prime}\right)^{\alpha p / 2}, \\
& E\left[\int_{0}^{T}\left(\left|p_{2}(t)-p_{2}^{\prime}(t)\right|^{2}+\left|Q_{2}(t)-Q_{2}^{\prime}(t)\right|^{2}\right) d t\right] \leq C_{1} d\left(u, u^{\prime}\right)^{\alpha p / 2} .
\end{aligned}
$$

The proof uses usual arguments from the theory of backward stochastic differential equations (see [26] for the details).

4.2.2. Necessary conditions for near optimality. For each $(t, x, a, p, q) \in[0,1] \times \mathbb{R}^{d} \times A$ $\times \mathbb{R}^{d} \times \mathbb{R}^{d \times d}$ define the Hamiltonian of the system

$$
H(t, x, a, p, q)=-h(t, x, a)-p \cdot b(t, x, a)-q \cdot \sigma(t, x, a) .
$$

Moreover we define the $\mathscr{H}$-function associated with a strict control $u(\cdot)$ and its corresponding trajectory $x(\cdot)$ by

$\mathscr{H}^{(x(\cdot), u(\cdot))}(t, x, a)=H\left(t, x, a, p_{1}(t), Q_{1}(t)-p_{2}(t) \cdot \sigma(t, x, u(t))\right)-\frac{1}{2} \sigma^{*}(t, x, a) p_{2}(t) \sigma(t, x, a)$,

where $(t, x, a) \in[0,1] \times \mathbb{R}^{d} \times A$ and $\left(p_{1}(t), Q_{1}(t)\right),\left(p_{2}(t), Q_{2}(t)\right)$ are solutions of the adjoint (4.16).

The next proposition gives necessary conditions for near optimality satisfied by the minimizing sequence $\left(u^{n}\right)$ (i.e., $\left(\mu^{n}\right)=\left(d t \delta_{u_{t}^{n}}(d a)\right)$ that converges to the optimal relaxed control $\left.d t \mu_{t}(d a)\right)$.

Proposition 4.5. Let $u^{n}$ be an admissible strict control such that

$$
J\left(u^{n}\right)=J\left(\mu^{n}\right) \leq \inf \{J(\mu) ; \mu \in \mathscr{R}\}+\epsilon_{n},
$$


then the following inequality holds:

$$
E\left(\int_{0}^{1} \mathscr{H}^{\left(x^{n}(t), u^{n}(t)\right)}\left(t, x^{n}(t), u^{n}(t)\right) d t\right) \geq \sup _{a \in A} E\left(\int_{0}^{1} \mathscr{H}^{\left(x^{n}(t), u^{n}(t)\right)}\left(t, x^{n}(t), a\right) d t\right)-\varepsilon^{1 / 3} .
$$

Sketch of the proof. According to Lemma 4.3, the cost functional $J(u)$ is continuous with respect to the topology induced by the metric $d$. Then by applying Ekeland's variational principle for $u^{n}$ with $\lambda=\varepsilon^{2 / 3}$, there exists an admissible control $\tilde{u}^{n}$ such that

$$
\begin{gathered}
d\left(u^{n}, \tilde{u}^{n}\right) \leq \varepsilon^{2 / 3}, \\
\bar{J}\left(\tilde{u}^{n}\right) \leq \bar{J}(u) \quad \forall u \in \mathcal{u}, \\
\bar{J}(u)=J(u)+\varepsilon^{1 / 3} d\left(u, \tilde{u}^{n}\right) .
\end{gathered}
$$

The control $v_{n}$ which is $\varepsilon_{n}$-optimal is in fact optimal for the new cost functional $\bar{J}(u)$. We proceed as in the classical maximum principle (Peng [22], Bensoussan [3]) to derive a maximum principle for $\tilde{u}^{n}(\cdot)$. Let $t_{0} \in(0,1), a \in A$; we define the strong variation by

$$
\tilde{u}_{\delta}^{n}= \begin{cases}a & \text { on }\left(t_{0}, t_{0}+\delta\right) \\ v_{n}(t) & \text { otherwise. }\end{cases}
$$

The fact that $\bar{J}\left(v^{n}\right) \leq \bar{J}\left(\tilde{u}^{n}\right)$ and $d\left(\tilde{u}^{n}, \tilde{u}_{\delta}^{n}\right) \leq \delta$ imply that

$$
J\left(\tilde{u}_{\delta}^{n}\right)-J\left(\tilde{u}^{n}\right) \geq-\varepsilon_{n}^{1 / 3} \delta .
$$

Proceeding as in Peng [22] (see also [26]) and using the smoothness of the data defining the control problem, we can expand $\tilde{X}_{\delta}^{n}(\cdot)$ (the solution of $(2.1)$ corresponding to $\tilde{u}_{\delta}^{n}$ ) to the second order to get the following inequality:

$$
\begin{aligned}
E \int_{t_{0}}^{t_{0}+\delta}\{ & \frac{1}{2}\left(\sigma\left(t, \tilde{X}^{n}(t), a\right)-\sigma\left(t, \tilde{X}^{n}(t), \tilde{u}^{n}\right)\right)^{*} \tilde{p}_{2}^{n}\left(\sigma\left(t, \tilde{X}^{n}(t), a\right)-\sigma\left(t, \tilde{X}^{n}(t), \tilde{u}^{n}\right)\right) \\
& +\tilde{p}_{1}^{n}\left(b\left(t, \tilde{X}^{n}(t), a\right)-b\left(t, \tilde{X}^{n}(t), \tilde{u}^{n}\right)\right)+\widetilde{Q}_{1}^{n}\left(\sigma\left(t, \tilde{X}^{n}(t), a\right)-\sigma\left(t, \tilde{X}^{n}(t), \tilde{u}^{n}\right)\right) \\
& \left.+\left(h\left(t, \tilde{X}^{n}(t), a\right)-h\left(t, \tilde{X}^{n}(t), \tilde{u}^{n}\right)\right)\right\} d t+o(\delta) \geq-\varepsilon_{n} \delta,
\end{aligned}
$$

where $\left(\tilde{p}_{1}^{n}, \widetilde{Q}_{1}^{n}\right)$ and $\left(\tilde{p}_{2}^{n}, \widetilde{Q}_{2}^{n}\right)$ are the first- and second-order adjoint processes, solutions of $(4.16)$ corresponding to $\left(\tilde{u}^{n}, \tilde{X}^{n}\right)$.

The variational inequality is obtained for $\tilde{u}^{n}$ by dividing by $\delta$ and tending $\delta$ to 0 .

The same claim can be proved for $u^{n}$ by using the stability of the state equations and the adjoint processes with respect to the control variable (Lemmas 4.1 and 4.4). 
Remark 4.6. The variational inequality (4.22) can be proved with the supremum over $a \in A$ replaced by the supremum over $u(\cdot) \in \mathcal{U}$ by simply putting $u(t)$ in place of $a$ in the definition of the strong perturbation.

4.3. The relaxed maximum principle. Assume that $\mathscr{F}_{t}$ is the natural filtration of the Brownian motion $B_{t}$. Let $\mu$ be a relaxed control and $x(\cdot)$ the corresponding trajectory. Let $\left(p_{1}, Q_{1}\right)$ and $\left(p_{2}, Q_{2}\right)$ be the solutions of the first- and second-order adjoint equations, associated with the optimal relaxed pair $(\mu, x)$,

$$
\begin{aligned}
& d p_{1}(t)=-[ b_{x}^{*}(t, x(t), \mu(t)) p_{1}(t)+\sigma_{x}^{*}(t, x(t), \mu(t)) Q_{1}(t) \\
&\left.+h_{x}(t, x(t), \mu(t))\right] d t+Q_{1}(t) d B_{t}, \\
& p_{1}(T)=g_{x}(x(T)), \\
&-d p_{2}(t)=-[ b_{x}^{*}(t, x(t), \mu(t)) p_{2}(t)+p_{2}(t) b_{x}(t, x(t), \mu(t)) \\
&+ \sigma_{x}^{*}(t, x(t), \mu(t)) p_{2}(t) \sigma_{x}(t, x(t), \mu(t)) \\
&+ \sigma_{x}^{*}(t, x(t), \mu(t)) Q_{2}(t) \\
&+\left.Q_{2}(t) \sigma_{x}\left(t, x(t), \mu(t)-H_{x x}\left(x(t), \mu(t), p_{1}(t), Q_{1}(t)\right)\right)\right] d t \\
&+ Q_{2}(t) d B_{t}, \\
& p_{2}(T)=g_{x x}(x(T)),
\end{aligned}
$$

where the notation $f(t, x(t), \mu(t))=\int_{A} f(t, x(t), a) \mu(t, d a)$, and $f$ stands for $b_{x}, \sigma_{x}, h_{x}$, and $H_{x x}$.

Define the function associated with the optimal pair $(\mu, x(\cdot))$ and their corresponding adjoint processes,

$$
\mathscr{H}^{(x(\cdot), \mu(\cdot))}(t, x, a)=H\left(t, x, a, p_{1}(t), Q_{1}(t)-p_{2}(t) \cdot \sigma(t, x, \mu(t))\right)-\frac{1}{2} \sigma^{*}(t, x, a) p_{2}(t) \sigma(t, x, a) .
$$

Theorem 4.7 (maximum principle). Let $(\mu, x)$ be an optimal relaxed pair, then

$$
E\left(\int_{0}^{1} \mathscr{H}^{(x(t), \mu(t))}(t, x(t), \mu(t)) d t\right)=\sup _{a \in A} E\left(\int_{0}^{1} \mathscr{H}^{(x(t), \mu(t))}(t, x(t), a) d t\right)
$$

Corollary 4.8. Under the same conditions as in Theorem 4.7, it holds that

$$
E\left(\int_{0}^{1} \mathscr{H}^{(x(t), \mu(t))}(t, x(t), \mu(t)) d t\right)=\sup _{v \in \mathbb{P}(A)} \int_{0}^{1} E\left[\mathscr{H}^{(x(t), \mu(t))}(t, x(t), v)\right] d t,
$$

where $\mathscr{H}^{(x(t), \mu(t))}(t, x(t), v)=\int_{A} \mathscr{H}^{(x(t), \mu(t))}(t, x(t), a) d a$. 
Proof. The inequality

$$
\sup _{\mu \in \mathbb{P}(A)} \int_{0}^{1} E\left[\mathscr{H}^{(x(t), \mu(t))}(t, x(t), v)\right] d t \geq \sup _{a \in A} E\left(\int_{0}^{1} \mathscr{H}^{(x(t), \mu(t))}(t, x(t), a) d t\right)
$$

is obvious. Indeed it suffices to take $\mu=\delta_{u}$, where $u$ is any point of $A$. Now if $v \in \mathbb{P}(A)$ is a probability measure on $A$, then

$$
\int_{0}^{1} E\left[\mathscr{H}^{(x(t), \mu(t))}(t, x(t), v)\right] d t \in \operatorname{conv}\left\{E\left(\int_{0}^{1} \mathscr{H}^{(x(t), \mu(t))}(t, x(t), a) d t\right), a \in A\right\} .
$$

Hence, by using a result on convex analysis, it holds that

$$
\int_{0}^{1} E\left[\mathscr{H}^{(x(t), \mu(t))}(t, x(t), v)\right] d t \leq \sup _{u \in A} E\left(\int_{0}^{1} \mathscr{H}^{(x(t), \mu(t))}(t, x(t), a) d t\right) .
$$

Remark 4.9. Since $\mathbb{P}(A)$ is a subspace of $\mathbb{V}$ consisting of constant $($ in $(\omega, t))$ relaxed controls, then (4.29) may be replaced by

$$
E\left(\int_{0}^{1} \mathscr{H}^{(x(t), \mu(t))}(t, x(t), \mu(t)) d t\right)=\sup _{v \in \mathbb{V}} \int_{0}^{1} E\left[\mathscr{H}^{(x(t), \mu(t))}(t, x(t), v(t))\right] d t
$$

Corollary 4.10 (the Pontriagin relaxed maximum principle). If $(\hat{\mu}, \hat{x})$ denotes an optimal relaxed pair, then there exists a Lebesgue negligible subset $N$ such that, for any tnot in $N$,

$$
\mathscr{H}^{(x(t), \mu(t))}(t, x(t), \mu(t))=\sup _{v \in \mathbb{P}(A)} \mathscr{H}^{(x(t), \mu(t))}(t, x(t), v), \quad P \text {-a.s. }
$$

Proof. Let $\theta \in] 0, T\left[\right.$ and $B \in \mathscr{F}_{\theta}$, for small $h>0$, define the relaxed control

$$
\mu_{t}^{h}= \begin{cases}v 1_{B} & \text { for } \theta<t<\theta+h \\ \widehat{\mu}_{t} & \text { otherwise }\end{cases}
$$

where $v$ is a probability measure on $A$. It follows from (4.29) that

$$
\frac{1}{h} \int_{\theta}^{\theta+h} E\left[1_{B} \mathscr{H}^{(x(t), \mu(t))}(t, x(t), \mu(t))\right] d t \geq \frac{1}{h} \int_{\theta}^{\theta+h} E\left[1_{B} \mathscr{H}^{(x(t), \mu(t))}(t, x(t), v)\right] d t .
$$

Therefore passing at the limit as $h$ tends to zero, we obtain

$$
E\left[1_{B} \int_{A} H\left(\theta, \hat{x}_{\theta}, a, p_{\theta}\right) \cdot \hat{\mu}_{\theta}(d a)\right] \geq E\left[1_{B} \int_{A} H\left(\theta, \hat{x}_{\theta}, u, p_{\theta}\right) \mu(d a)\right]
$$

for any $\theta$ not in some Lebesgue null set $N$.

The last inequality is valid for all $B \in \mathscr{F}_{\theta}$, then for any bounded $\mathscr{F}_{\theta}$-measurable random variable $F$, it holds that

$$
E\left[F_{\mathcal{H}^{(x(t), \mu(t))}}(t, x(t), \mu(t))\right] \geq E\left[F^{\mathcal{H}^{(x(t), \mu(t))}}(t, x(t), v)\right],
$$


which leads to

$$
E\left[\mathscr{H}^{(x(\theta), \mu(\theta))}(\theta, x(\theta), \mu(\theta)) / \mathscr{F}_{\theta}\right] \geq E\left[\mathscr{H}^{(x(\theta), \mu(\theta))}(\theta, x(\theta), v) / \mathscr{F}_{\theta}\right] .
$$

The result follows from the measurability with respect to $\mathscr{F}_{\theta}$ of the quantities inside the conditional expectation.

The proof of Theorem 4.7 is based on the next lemma on the passage to the limit as $n$ tends to $+\infty$ in the adjoint processes $\left(p_{1}^{n}, Q_{1}^{n}\right)$ and $\left(p_{2}^{n}, Q_{2}^{n}\right)$ as well as in the variational inequality (4.22).

Lemma 4.11. Let $\left(p_{1}^{n}, Q_{1}^{n}\right)$ and $\left(p_{2}^{n}, Q_{2}^{n}\right)$ (resp., $\left(p_{1}, Q_{1}\right)$ and $\left.\left(p_{2}, Q_{2}\right)\right)$ be defined by (4.16) associated with the pair $\left(u^{n}, x^{n}\right)$, (resp., (4.27)), then it holds that

(i) $\lim _{n \rightarrow+\infty} E\left[\int_{0}^{T}\left(\left|p_{1}(t)-p_{1}^{n}(t)\right|^{2}+\left|Q_{1}(t)-Q_{1}^{n}(t)\right|^{2}\right) d t\right]=0$;

(ii) $\lim _{n \rightarrow+\infty} E\left[\int_{0}^{T}\left(\left|p_{2}(t)-p_{2}^{n}(t)\right|^{2}+\left|Q_{2}(t)-Q_{2}^{n}(t)\right|^{2}\right) d t\right]=0$;

(iii) $\lim _{n \rightarrow+\infty} E\left(\int_{0}^{1} \mathscr{H}^{\left(x^{n}(t), u^{n}(t)\right)}\left(t, x^{n}(t), u^{n}(t)\right) d t\right)=E\left(\int_{0}^{1} \mathscr{H}^{(x(t), \mu(t))}(t, x(t), \mu(t)) d t\right)$.

Proof. Let us prove (i).

For simplicity of notations, we denote by

$$
\begin{aligned}
A_{n}(t)=b_{x}^{*}\left(t, x^{n}(t), u^{n}(t)\right), & A(t)=b_{x}^{*}(t, x(t), \mu(t)), \\
B_{n}(t)=\sigma_{x}^{*}\left(t, x^{n}(t), u^{n}(t)\right), & B(t)=\sigma_{x}^{*}(t, x(t), \mu(t)), \\
C_{n}(t)=h_{x}^{*}\left(t, x^{n}(t), u^{n}(t)\right), & C(t)=h_{x}^{*}(t, x(t), \mu(t)), \\
f_{n}(t, p, Q)=A_{n}(t) p+B_{n}(t) Q+C_{n}(t), & f(t, p, Q)=A(t) p+B(t) Q+C(t), \\
\xi_{n}=g_{x}\left(x^{n}(T)\right), & \xi=g_{x}(x(T)) .
\end{aligned}
$$

$g_{x}$ being Lipschitz continuous and $x^{n}(T)$ converging in probability to $x(T)$, hence $\xi_{n}$ converges to $\xi$. Then without loss of generality we may suppose that $\xi_{n}=\xi$.

Applying Ito's formula for $\left|p_{1}(t)-p_{1}^{n}(t)\right|^{2}$, it holds that

$$
\begin{aligned}
\mid p_{1}(t) & -\left.p_{1}^{n}(t)\right|^{2}+\int_{t}^{1}\left|Q_{1}(s)-Q_{1}^{n}(s)\right|^{2} d s \\
= & 2 \int_{t}^{1}\left(p_{1}(s)-p_{1}^{n}(s)\right)^{*}\left(f_{n}\left(s, p_{1}^{n}(s), Q_{1}^{n}(s)\right)-f\left(s, p_{1}(s), Q_{1}(s)\right)\right) d s \\
& -\int_{t}^{1}\left(p_{1}(s)-p_{1}^{n}(s)\right)^{*}\left(Q_{1}(s)-Q_{1}^{n}(s)\right) d B_{s},
\end{aligned}
$$

where $\left(p_{1}(t)-p_{1}^{n}(t)\right) *$ denotes the transpose of $\left(p_{1}(t)-p_{1}^{n}(t)\right)$. 
Let $\alpha$ be an arbitrary real positive number. By using Young inequality and taking expectations in both sides, we get

$$
\begin{aligned}
E\left(\left|p_{1}(t)-p_{1}^{n}(t)\right|^{2}+\int_{t}^{1}\left|Q_{1}(s)-Q_{1}^{n}(s)\right|^{2}\right) & \\
\leq & 2 E \int_{t}^{1}\left|p_{1}(s)-p_{1}^{n}(s)\right|\left|f_{n}\left(s, p_{1}^{n}(s), Q_{1}^{n}(s)\right)-f\left(s, p_{1}(s), Q_{1}(s)\right)\right| d s \\
\leq & 2 \alpha^{2} E \int_{t}^{1}\left|p_{1}(s)-p_{1}^{n}(s)\right|^{2} d s+\frac{2}{\alpha^{2}} E \int_{t}^{1}\left|f_{n}\left(s, p_{1}^{n}(s), Q_{1}^{n}(s)\right)-f\left(s, p_{1}(s), Q_{1}(s)\right)\right|^{2} d s \\
\leq & 2 \alpha^{2} E \int_{t}^{1}\left|p_{1}(s)-p_{1}^{n}(s)\right|^{2} d s \\
& +\frac{4}{\alpha^{2}} E \int_{t}^{1}\left|f_{n}\left(t, p_{1}^{n}(t), Q_{1}^{n}(t)\right)-f_{n}\left(t, p_{1}, Q_{1}\right)\right|^{2} d s \\
& +\frac{4}{\alpha^{2}} E \int_{t}^{1}\left|f_{n}\left(t, p_{1}, Q_{1}\right)-f\left(t, p_{1}, Q_{1}\right)\right|^{2} d s \\
\leq & 2 \alpha^{2} E \int_{t}^{1}\left|p_{1}(t)-p_{1}^{n}(t)\right|^{2} d s \\
& +\frac{4}{\alpha^{2}}\left\{E \int_{t}^{1}\left|A_{n}(t)\right|^{2}\left|p_{1}(t)-p_{1}^{n}(t)\right|^{2} d s+E \int_{t}^{1}\left|B_{n}(t)\right|^{2}\left|Q_{1}(t)-Q_{1}^{n}(t)\right|^{2} d s\right\} \\
& +\frac{4}{\alpha^{2}}\left\{\int_{t}^{1}\left|A_{n}(t)-A(t)\right|^{2}\left|p_{1}(t)\right|^{2} d s+E \int_{t}^{1}\left|B_{n}(t)-B(t)\right|^{2}\left|Q_{1}(t)\right|^{2} d s\right.
\end{aligned}
$$

$A_{n}(t)$ and $B_{n}(t)$ are uniformly bounded by the common Lipshitz constant $C$ of $b$ and $\sigma$.

We choose $\alpha$ such that $4 C^{2} / \alpha^{2}<1$, and use Gronwall lemma to get

$$
\begin{aligned}
& E\left(\left|p_{1}(t)-p_{1}^{n}(t)\right|^{2}+\int_{t}^{1}\left|Q_{1}(t)-Q_{1}^{n}(t)\right|^{2}\right) \\
& \leq M\left\{E \int_{t}^{1}\left|A_{n}(t)-A(t)\right|^{2}\left|p_{1}(t)\right|^{2} d s+E \int_{t}^{1}\left|B_{n}(t)-B(t)\right|^{2}\left|Q_{1}(t)\right|^{2} d s\right. \\
& \left.+E \int_{t}^{1}\left|C_{n}(t)-C(t)\right|^{2} d s\right\} .
\end{aligned}
$$

The result follows from the fact that $A_{n}(t), B_{n}(t), C_{n}(t)$ converge to $A(t), B(t)$, and $C(t)$. 
To get the result with sup norm, it is sufficient to use Burkholder-Davis-Gundy inequality to the martingale part in Ito's formula.

(ii) and (iii) are proved by using the same arguments.

Proof of Theorem 4.7. The result is proved by passing to the limit in inequality (4.22) and using Lemma 4.11.

\section{Appendix}

Lemma A.1 (Skorokhod selection theorem [11, page 9]). Let $(S, \rho)$ be a complete separable metric space, and let $P$ and $P_{n}, n=1,2, \ldots$, be probability measures on $\left(S, B_{S}\right)$ such that $\left(P_{n}\right)$ converges weakly to $P$. Then, on a probability space $\left(\widetilde{\Omega}, \widetilde{F_{F}}, \widetilde{P}\right)$, there exist $S$-valued random variables $X^{n}, n=1,2, \ldots$, and $X$ such that

(1) $P=\widetilde{P}_{X}$;

(2) $P_{n}=\widetilde{P}_{X^{n}}, n=1,2, \ldots$;

(3) $X^{n} \underset{n \rightarrow \infty}{\longrightarrow} X \widetilde{P}-$ a.s.

Lemma A.2 (Kolmogorov's theorem [11, page 18]). Let $\left(X_{t}^{n}\right)_{n \geq 0}$ be a sequence of d-dimensional continuous processes satisfying the following two conditions:

(i) there exist positive constants $m$ and $\gamma$ such that $E\left[\left|X_{0}^{n}\right|^{\gamma}\right] \leq m, n \geq 0$;

(ii) there exist positive constants $\alpha, \beta, m_{k}, k=1,2, \ldots$, such that

$$
E\left[\left|X_{t}^{n}-X_{s}^{n}\right|^{\alpha}\right] \leq m_{k}|t-s|^{1+\beta}, \quad n \geq 0 \forall t, s \in[0, k], k=1,2, \ldots .
$$

Then there exist a subsequence $\left(n_{k}\right)$, a probability space $\left(\widetilde{\Omega}, \widetilde{F_{F}}, \widetilde{P}\right)$, and d-dimensional continuous processes $\tilde{X}^{n_{k}}, k=1,2, \ldots$, and $\tilde{X}$, defined on it such that

(1) the laws of $\tilde{X}^{n_{k}}$ and $X^{n_{k}}$ coincide;

(2) $\tilde{X}_{t}^{n_{k}}$ converges to $\tilde{X}_{t}$ uniformly on every finite time interval, $\widetilde{P}-a . s$.

Lemma A.3 (Mitoma [20]). Let $C_{E^{\prime}}=C\left([0,1], E^{\prime}\right)$ be the space of continuous mappings from $[0,1]$ to $E^{\prime}$ the topological dual of some Frêchet space E. Let $\left(P_{n}\right)$ be a sequence of probability measures on $C_{E^{\prime}}$. Suppose that for each $\xi$ in $E$, the sequence $\left(P_{n} \Pi_{\xi}^{-1}\right)$ is tight in $C$, where $\Pi_{\xi}: x \in C_{E^{\prime}} \rightarrow\langle x ., \xi\rangle \in C=C\left([0,1], \mathbb{R}^{d}\right)$. Then the sequence $\left(P_{n}\right)$ itself is tight.

\section{Acknowledgment}

This work is supported by MENA Swedish-Algerian Research Partnership Program (3482002-6874).

\section{References}

[1] K. Bahlali, B. Mezerdi, and Y. Ouknine, Pathwise uniqueness and approximation of solutions of stochastic differential equations, Séminaire de Probabilités, XXXII, Lecture Notes in Math., vol. 1686, Springer, Berlin, 1998, pp. 166-187.

[2] H. Becker and V. Mandrekar, On the existence of optimal random controls, Journal of Mathematics and Mechanics 18 (1969), no. 12, 1151-1166.

[3] A. Bensoussan, Lectures on stochastic control, Nonlinear Filtering and Stochastic Control, Lecture Notes in Math., vol. 972, Springer, Berlin, 1982, pp. 1-62. 
[4] N. El Karoui and S. Méléard, Martingale measures and stochastic calculus, Probability Theory and Related Fields 84 (1990), no. 1, 83-101.

[5] N. El Karoui, D. H. Nguyen, and M. Jeanblanc-Picqué, Compactification methods in the control of degenerate diffusions: existence of an optimal control, Stochastics 20 (1987), no. 3, 169-219.

[6] __ Existence of an optimal Markovian filter for the control under partial observations, SIAM Journal on Control and Optimization 26 (1988), no. 5, 1025-1061.

[7] W. H. Fleming, Generalized solutions in optimal stochastic control, Differential Games and Control Theory, II (Proceedings of 2nd Conference, University of Rhode Island, Kingston, RI, 1976), Lecture Notes in Pure and Appl. Math., 30, Marcel Dekker, New York, 1977, pp. 147-165.

[8] A. Ghouila-Houri, Sur la généralisation de la notion de commande d'un système guidable, RAIRO. Recherche Opérationnelle 1 (1967), no. 4, 7-32.

[9] U. G. Haussmann, Existence of optimal Markovian controls for degenerate diffusions, Stochastic Differential Systems, Lecture Notes in Control and Inform. Sci., vol. 78, Springer, Berlin, 1986, pp. 171-186.

[10] U. G. Haussmann and J.-P. Lepeltier, On the existence of optimal controls, SIAM Journal on Control and Optimization 28 (1990), no. 4, 851-902.

[11] N. Ikeda and S. Watanabe, Stochastic Differential Equations and Diffusion Processes, NorthHolland Mathematical Library, vol. 24, North-Holland, Amsterdam, 1981.

[12] J. Jacod and J. Mémin, Sur un type de convergence intermédiaire entre la convergence en loi et la convergence en probabilité, Seminar on Probability, XV (University of Strasbourg, Strasbourg, 1979/1980) (French), Lecture Notes in Math., vol. 850, Springer, Berlin, 1981, pp. 529-546.

[13] H. J. Kushner, Existence results for optimal stochastic controls, Journal of Optimization Theory and Applications 15 (1975), 347-359.

[14] S. Méléard, Martingale measure approximation, application to the control of diffusions, Prépublication 69, Labo. Probabilités, Université de Paris VI, 1992.

[15] S. Méléard and S. Roelly-Coppoletta, Système de particules et mesures-martingales: un théorème de propagation du chaos, Sèminaire de Probabilitès, Lecture Notes in Math., vol. 1321, Springer, Berlin, 1988, pp. 438-448.

[16] _ A generalized equation for a continuous measure branching process, Stochastic Partial Differential Equations and Applications, II (Trento, 1988), Lecture Notes in Math., vol. 1390, Springer, Berlin, 1989, pp. 171-185.

[17] B. Mezerdi, Necessary conditions for optimality for a diffusion with a nonsmooth drift, Stochastics 24 (1988), no. 4, 305-326.

[18] B. Mezerdi and S. Bahlali, Approximation in optimal control of diffusion processes, Random Operators and Stochastic Equations 8 (2000), no. 4, 365-372.

[19] _ Necessary conditions for optimality in relaxed stochastic control problems, Stochastics and Stochastics Reports 73 (2002), no. 3-4, 201-218.

[20] I. Mitoma, Tightness of probabilities on $C\left([0,1] ; \mathscr{Y}^{\prime}\right)$ and $D\left([0,1] ; \mathscr{Y}^{\prime}\right)$, The Annals of Probability 11 (1983), no. 4, 989-999.

[21] É. Pardoux and S. G. Peng, Adapted solution of a backward stochastic differential equation, Systems \& Control Letters 14 (1990), no. 1, 55-61.

[22] S. G. Peng, A general stochastic maximum principle for optimal control problems, SIAM Journal on Control and Optimization 28 (1990), no. 4, 966-979.

[23] A. V. Skorokhod, Studies in the Theory of Random Processes, Addison-Wesley, Massachusetts, 1965. 
[24] J. B. Walsh, An introduction to stochastic partial differential equations, École d'été de Probabilités de Saint-Flour, XIV—1984, Lecture Notes in Math., vol. 1180, Springer, Berlin, 1986, pp. 265439.

[25] J. Yong and X. Y. Zhou, Stochastic Controls, Stochastic Controls, Hamiltonian Systems and HJB Equations, vol. 43, Springer, New York, 1999.

[26] X. Y. Zhou, Stochastic near-optimal controls: necessary and sufficient conditions for near-optimality, SIAM Journal on Control and Optimization 36 (1998), no. 3, 929-947.

Seïd Bahlali: Laboratory of Applied Mathematics, University of Biskra, P.O. Box 145,

Biskra 07000, Algeria

E-mail address: sbahlali@yahoo.fr

Brahim Mezerdi: Laboratory of Applied Mathematics, University of Biskra, P.O. Box 145, Biskra 07000, Algeria

E-mail address: bmezerdi@yahoo.fr

Boualem Djehiche: Department of Mathematics, Division of Mathematical Statistics, Royal Institute of Technology, 10044 Stockholm, Sweden

E-mail address: boualem@math.kth.se 


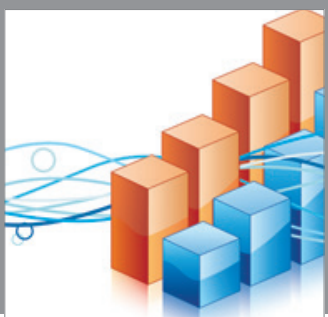

Advances in

Operations Research

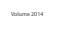

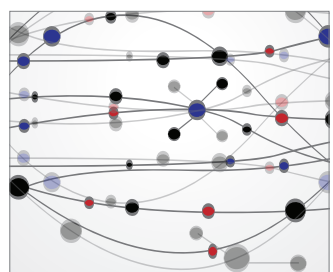

\section{The Scientific} World Journal
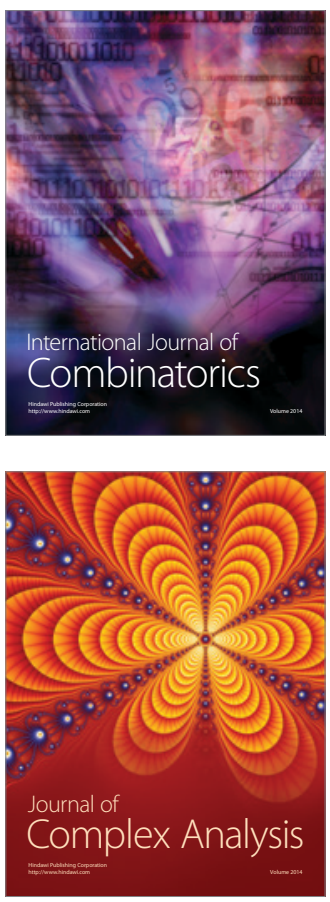

International Journal of

Mathematics and

Mathematical

Sciences
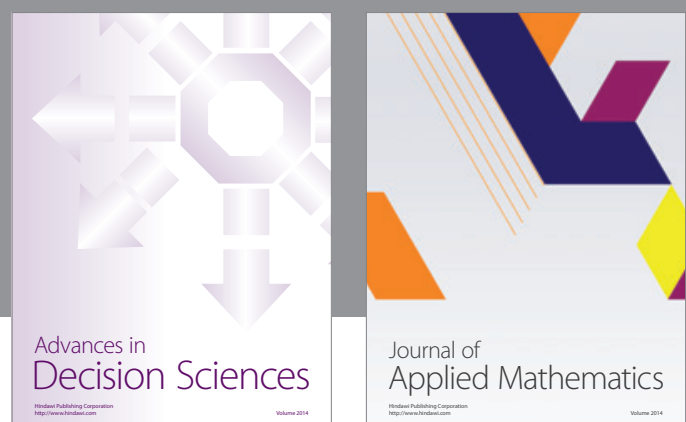

Journal of

Applied Mathematics
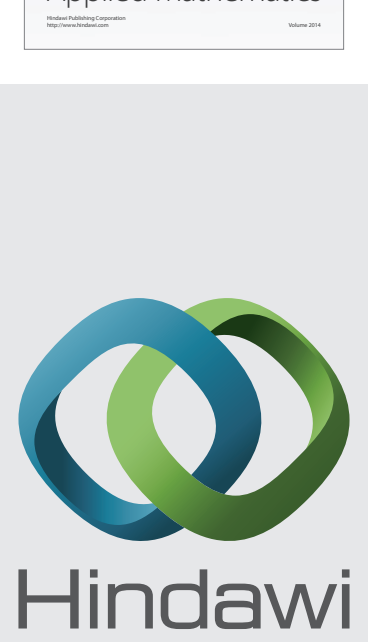

Submit your manuscripts at http://www.hindawi.com
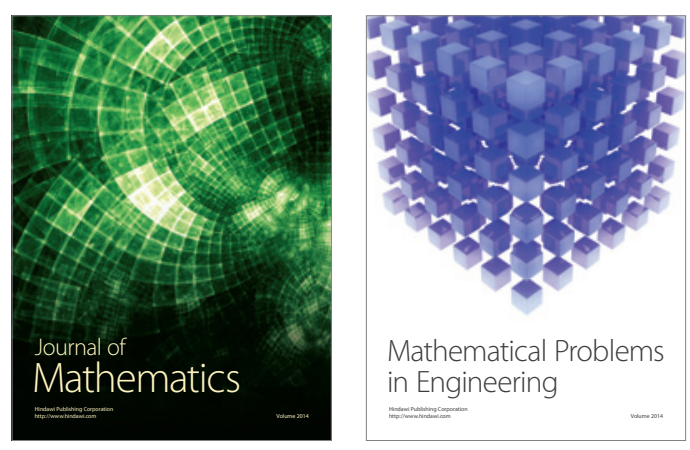

Mathematical Problems in Engineering
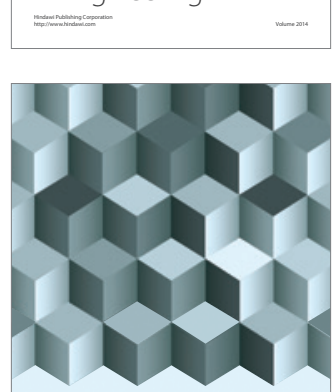

Journal of

Function Spaces
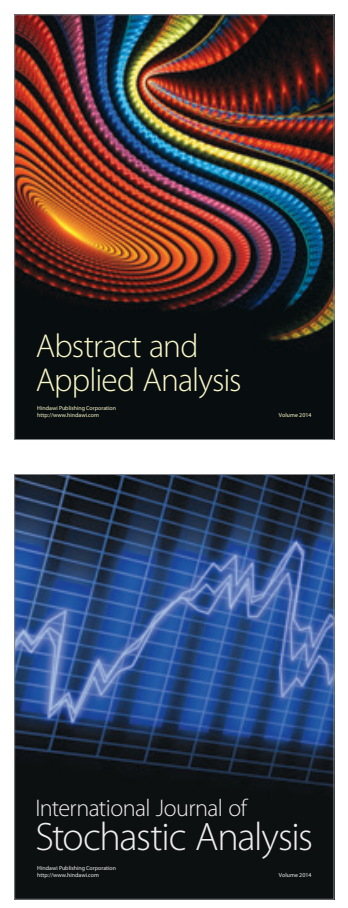

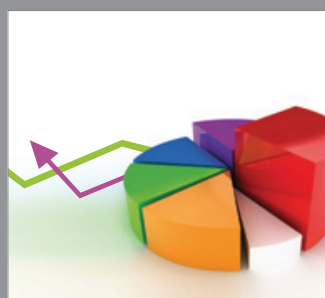

ournal of

Probability and Statistics

Promensencen
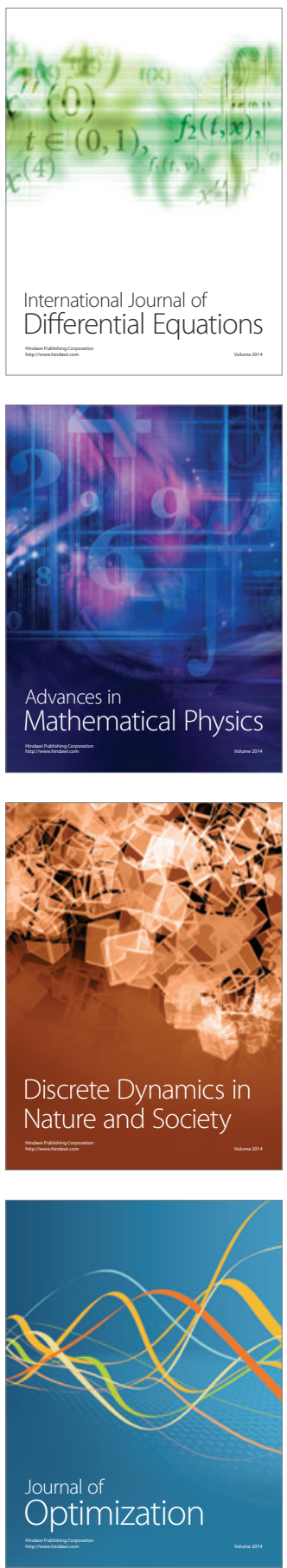